\title{
The metabolic effects of a commercially available chicken peri-peri (African bird's eye chilli) meal in overweight individuals
}

\author{
Jacolene Kroff, David J. Hume, Paula Pienaar, Ross Tucker, Estelle V. Lambert and Dale E. Rae* \\ UCT/MRC Research Unit for Exercise Science and Sports Medicine, Department of Human Biology, Faculty of Health Sciences, \\ University of Cape Town, 7700 Cape Town, South Africa
}

(Submitted 14 November 2013 - Final revision received 3 July 2015 - Accepted 14 July 2015 - First published online 11 September 2015)

\begin{abstract}
A growing body of evidence suggests that capsaicin ingestion may lead to desirable metabolic outcomes; however, the results in humans are equivocal. Whether or not benefits may be gained from ingestion of capsaicin via a commercially available meal has not been determined. The objectives of this randomised, cross-over intervention study were to compare the $2 \mathrm{~h}$ postprandial effects of a standard commercially prepared meal containing chilli (HOT, $5.82 \mathrm{mg}$ total capsaicinoids) with a similar meal with no chilli (CON, $<1.0 \mathrm{mg}$ total capsaicinoids) on resting energy expenditure, plasma insulin, glucose, serum high sensitivity C-reactive protein (hs-CRP) concentrations, core body temperature and forearm microvascular reactivity responses in overweight individuals. A total of thirty-four apparently healthy individuals (sixteen men and eighteen women) between 18 and 50 years of age, with a BMI $>25 \mathrm{~kg} / \mathrm{m}^{2}$ and a waist circumference $>94 \mathrm{~cm}$ (men) or $80 \mathrm{~cm}$ (women), were studied. Participants had normal glucose tolerance and were accustomed, but were not regular chilli eaters. A paired $t$ test indicated that insulin AUC was smaller following the HOT meal $(P=0 \cdot 002)$. Similarly, there was a tendency for glucose AUC to be reduced following the HOT meal $(P=0.056)$. No discernable effects of the HOT meal were observed on metabolic rate, core temperature, hs-CRP concentrations and endothelial-dependent microvascular reactivity. The results from this study indicate that a standard restaurant meal containing a relatively small dose of capsaicin delivered via African bird's eye chilli, which is currently available to the public, results in lower postprandial insulin concentrations in overweight individuals, compared with the same meal without chilli.
\end{abstract}

Key words: Capsaicin: Postprandial glucose: Insulin responses

Numerous studies have investigated the potential health benefits of specific food types and culinary ingredients. These include purported improvements in markers of health following ingestion of foods and beverages containing caffeine ${ }^{(1)}$, grains ${ }^{(2)}, \operatorname{cocoa}^{(3,4)}$ and flavonoids ${ }^{(5,6)}$. The consumption of chilli-containing foods has also been a focus of interest. Specifically, capsaicin (8-methyl$\mathrm{N}$-vanillyl-6-nonenamide), a member of the capsaicinoid family and the component of chilli responsible for its spicy heat (pungency), has been investigated ${ }^{(7-13)}$.

A growing body of evidence suggests that capsaicin ingestion may lead to desirable metabolic outcomes such as increased metabolic rate and fat oxidation, and improved digestive function. For example, early research found that capsaicin consumption reduced overall adiposity in rats, potentially via altered sympathetic nervous system function ${ }^{(14)}$, which enhanced lipolysis and metabolic rate ${ }^{(15)}$. More recently, dietary capsaicin was found to reduce obesity-induced glucose intolerance in rats, not only by suppressing inflammatory responses but also by enhancing fatty acid oxidation in adipose tissue and/or the liver, both of which are important peripheral tissues affecting insulin resistance ${ }^{(16)}$. Another study in rats found that ingestion of capsaicin reduced metabolic dysregulation in obese/diabetic mice by reducing fasting glucose, insulin and TAG concentrations ${ }^{(17)}$.

In human studies, the metabolic effects of capsaicin ingestion have been equivocal. Some studies have found little to no effect of capsaicin ingestion on metabolic rate or substrate utilisation ${ }^{(1,8,18-20)}$, whereas others have shown an effect $^{(2,9,11,12,18,19,21-24)}$. Differences in study design, which may partly account for these disparate findings, include method of ingestion (i.e. capsule $v$. chilli in a meal), active ingredient used (i.e. capsinoid $v$. capsaicinoid) and population studied (i.e. habitual $v$. non-habitual chilli eaters or normal weight $v$. overweight). Interestingly, the largest positive postprandial effects of a chilli-containing meal on resting energy expenditure, fuel oxidation and insulin response have been observed in participants with a BMI $>25 \mathrm{~kg} / \mathrm{m}^{2(3,4,11,25)}$. Current findings relating to the effects of chilli ingestion on metabolic rate, core temperature and endothelial function are also controversial.

Abbreviations: ACh, acetylcholine; CON, control meal; HOT, intervention meal; hs-CRP, high sensitivity C-reactive protein; VR, endothelial-dependent micro-vascular reactivity.

* Corresponding author: Dr D. E. Rae, fax +27 21650 1796, email dale.rae@uct.ac.za 
For example, some studies have shown that capsaicin ingestion increases metabolic rate and lipid oxidation ${ }^{(5,6,19,24,26)}$, induces a sweating response ${ }^{(7-13,22)}$, increases satiety and reduces energy and fat intake ${ }^{(14,27)}$, and stimulates carbohydrate oxidation at rest and during exercise ${ }^{(15,23)}$. Others have failed to demonstrate these effects $^{(8,11-13,16)}$.

In particular, the regulation of postprandial glucose concentrations may have clinical significance for overweight individuals with early-stage insulin resistance, a symptom of the metabolic syndrome associated with endothelial dysfunction ${ }^{(17,28,29)}$ and a precursor to diabetes ${ }^{(30,31)}$. Whether or not potential benefits may be gained from ingestion of capsaicin in the context of a standard commercially prepared meal has not yet been determined. Therefore, we set out to investigate whether a commercially available meal containing capsaicin, specifically from the African bird's eye chilli, improves postprandial glucose and insulin concentrations in overweight and obese individuals, who are at increased risk for insulin resistance.

The aim of this study was to measure the metabolic sequelae following the ingestion of a commercially available chillicontaining meal in overweight individuals. Specifically, the objectives were to compare the postprandial effects of the intervention and control meals on resting energy expenditure, plasma insulin, glucose, serum high sensitivity C-reactive protein (hs-CRP) concentrations, core body temperature and forearm microvascular reactivity responses in overweight individuals. We hypothesised that the commercially available capsaicin-containing meal would increase postprandial energy expenditure and improve insulin sensitivity compared with the control meal in our participants, and that these changes would be accompanied by increased thermogenic and inflammatory responses.

\section{Methods}

\section{Participants}

A total of forty overweight but apparently healthy individuals (eighteen men and twenty-two women) were recruited via advertisements placed in the print media. Participants were included in the study if they were between 18 and 50 years of age, had a waist circumference $>94 \mathrm{~cm}$ (men) or $80 \mathrm{~cm}$ (women), together with a BMI $>25 \mathrm{~kg} / \mathrm{m}^{2}$, and had normal glucose tolerance (plasma glucose value $2 \mathrm{~h}$ post-glucose tolerance test $\left.<7.8 \mathrm{mmol} / \mathrm{l}^{(32)}\right)$. In addition, participants needed to be accustomed to eating chilli, but may not have consumed chilli more than twice per week during the 3 months prior to testing. One male was excluded because his BMI $\left(64.2 \mathrm{~kg} / \mathrm{m}^{2}\right)$ and RMR (14 $133 \mathrm{~kJ} / \mathrm{d})$ were both >2 sD above the group mean. Two participants were subsequently excluded when it became known that they were currently under treatment for chronic diseases. Three more women were excluded with abnormal glucose tolerance. These six participants were excluded after the data collected during visit 1 were analysed. The general characteristics of the remaining thirty-four participants are presented in Table 1.

\section{Study design}

Participants were required to visit the UCT/MRC Research Unit for Exercise Science and Sports Medicine (University of Cape Town) on three occasions during this randomised cross-over intervention trial, with approximately 1 week separating each visit. The first visit served to screen participants for eligibility and to familiarise them with the tests to be conducted during the subsequent two visits. The second and third visits constituted the intervention trials during which each participant's metabolic response to two commercially available chicken burger meals was assessed. On one occasion, they consumed a chilli-containing meal (HOT) and on the other a control meal (CON). The HOT meal contained capsaicin (from the African bird's eye chilli plant), whereas the CON meal contained only trace amounts of capsaicin.

Participants drew a number from a container to randomly determine the order in which the HOT and CON meals would be served during visits 2 and 3. If an uneven number was drawn, the CON meal was served during visit 2 (the first

Table 1. Descriptive characteristics of the participants based on data collected during the familiarisation visit (Mean values, standard deviations and ranges)

\begin{tabular}{|c|c|c|c|c|c|c|c|c|}
\hline & \multicolumn{3}{|c|}{ All (n 34) } & \multicolumn{2}{|c|}{ Males $(n 16)$} & \multicolumn{2}{|c|}{ Females (n 18) } & \multirow[b]{2}{*}{$P^{*}$} \\
\hline & Mean & Range & SD & Mean & SD & Mean & SD & \\
\hline Age (years) & $32 \cdot 8$ & $19-48$ & $7 \cdot 2$ & $32 \cdot 4$ & $6 \cdot 8$ & $33 \cdot 2$ & $7 \cdot 6$ & 0.737 \\
\hline Height (m) & 171.4 & $148-190$ & $10 \cdot 5$ & $180 \cdot 6$ & $5 \cdot 0$ & $163 \cdot 2$ & $6 \cdot 3$ & $<0.000$ \\
\hline Weight (kg) & $91 \cdot 2$ & $61-125$ & $14 \cdot 2$ & $95 \cdot 8$ & $11 \cdot 7$ & $87 \cdot 1$ & $15 \cdot 2$ & 0.075 \\
\hline BMI $\left(\mathrm{kg} / \mathrm{m}^{2}\right)$ & $31 \cdot 2$ & $25-43$ & $5 \cdot 0$ & $29 \cdot 3$ & $3 \cdot 1$ & $32 \cdot 8$ & 5.9 & 0.129 \\
\hline Waist circumference $(\mathrm{cm})$ & $97 \cdot 1$ & $82-118$ & $8 \cdot 7$ & $97 \cdot 8$ & $5 \cdot 3$ & $96 \cdot 4$ & $11 \cdot 0$ & 0.512 \\
\hline Body fat $(\%)$ & $34 \cdot 3$ & $17-51$ & $9 \cdot 1$ & $26 \cdot 8$ & 4.4 & $41 \cdot 0$ & $6 \cdot 6$ & $<0.000$ \\
\hline Fat-free mass (kg) & $59 \cdot 7$ & 40-90 & 11.9 & $70 \cdot 0$ & 8.5 & $50 \cdot 6$ & $4 \cdot 6$ & $<0.000$ \\
\hline Fasting glucose (mmol/l) & 4.95 & $4 \cdot 06-6 \cdot 13$ & 0.50 & 5.07 & 0.37 & 4.85 & 0.59 & 0.211 \\
\hline Fasting insulin (mU/l) & $10 \cdot 02$ & $4 \cdot 22-30 \cdot 83$ & 6.08 & $7 \cdot 47$ & $3 \cdot 18$ & $12 \cdot 29$ & $7 \cdot 17$ & 0.022 \\
\hline HOMA-IR & $2 \cdot 22$ & $0.8-6.54$ & 1.42 & 1.69 & 0.77 & $2 \cdot 70$ & 1.70 & 0.081 \\
\hline OGTT $120 \mathrm{~min}$ glucose $(\mathrm{mmol} / \mathrm{l})$ & $5 \cdot 90$ & $2 \cdot 99-7 \cdot 75$ & $1 \cdot 27$ & $5 \cdot 65$ & $1 \cdot 20$ & $6 \cdot 12$ & 1.33 & 0.298 \\
\hline OGTT $120 \mathrm{~min}$ insulin $(\mathrm{mU} / \mathrm{l})$ & $62 \cdot 89$ & $8 \cdot 3-159 \cdot 3$ & $47 \cdot 01$ & $47 \cdot 71$ & 43.67 & $76 \cdot 38$ & $46 \cdot 87$ & 0.067 \\
\hline Insulinogenic index & 1.96 & $0.25-5.48$ & $1 \cdot 30$ & 1.61 & $1 \cdot 10$ & $2 \cdot 27$ & 1.41 & 0.070 \\
\hline
\end{tabular}

HOMA-IR, homoeostasis model of assessment - insulin resistance; OGTT, oral glucose tolerance test.

* The $P$ value represents the comparison between the males and females determined using an independent $t$ test or Mann-Whitney $U$ test. 
meal-response test) and the HOT meal during visit 3 (the second meal-response test). If an even number was drawn, the HOT meal visit preceded the CON meal visit. The study was also counter-balanced so that half of the participants received the HOT meal during visit 2 and the other half during visit 3. As the HOT meal produces a distinctive taste and burning sensation in the mouth, it was not possible to blind participants to the type of meal being eaten. This study was conducted according to the guidelines laid down in the Declaration of Helsinki (October 2008, Seoul). Each participant signed an informed consent form prior to entering the trial. The Human Research Ethics Committee of the University of Cape Town approved the study (UCT HREC Ref. No. 373/2011).

\section{Detailed testing procedures}

Visit 1 (familiarisation). The volunteers reported to the laboratory between 07.00 and 09.00 hours following an overnight fast (10-12 h). The investigator measured the height, weight and waist circumference ${ }^{(33)}$ of the participants, and their body composition (fat mass, lean mass and total body water) was determined in the supine position using bioelectrical impedance (Quantum II body composition analyser; RJL System). Participants were then familiarised with the equipment and procedures to be used for the RMR (using an indirect calorimetry ventilated hood technique) and the endothelial microvascular reactivity (VR, using laser Doppler imaging and iontophoresis) tests to be conducted during subsequent visits. Lastly, participants underwent a $75 \mathrm{~g}$ oral glucose tolerance test (OGTT) for which fasting (0 min), 30, 60, 90 and $120 \mathrm{~min}$ venous blood samples were drawn for the determination of plasma glucose and serum insulin concentrations. Before leaving, the investigator gave the volunteers a CorTemp ${ }^{\mathrm{TM}}$ capsule to be ingested $11 \mathrm{~h}$ prior to visit 2 .

Visits 2 and 3. Visits 2 and 3 took place in a randomised, counter-balanced order and were identical except for the fact that on one occasion the participants ate the HOT meal, whereas on the other they ate the CON meal. Testing began between 07.00 and 09.00 hours, and the start time for visit 3 was matched to that of visit 2 . Prior to each visit, participants were asked to avoid alcohol, fried food and vigorous exercise for $24 \mathrm{~h}$, to fast for $10-12 \mathrm{~h}$ (i.e. overnight) and to ingest the CorTemp ${ }^{\mathrm{TM}}$ capsule at approximately 22.00 hours the preceding night. The order of testing is illustrated in Fig. 1. The investigator measured the weight of the participants, who then rested in the supine position for $10 \mathrm{~min}$. Baseline core body temperature, RMR (20 min in length) and VR (30 min in length) measurements were taken, following which an in-dwelling cannula was inserted into either an antecubital or a hand vein. Venous blood samples were drawn to measure fasting baseline glucose and insulin concentrations, as well as the anti-inflammatory marker, hs-CRP. Participants then had $10 \mathrm{~min}$ in which to consume either the HOT or the CON meal and were permitted to drink $250 \mathrm{ml}$ of water after the meal.

Participants underwent two postprandial RMR tests ( $0-40$ and 80-120 min post-meal) and a postprandial VR test (45-75 min post-meal) in the $2 \mathrm{~h}$ period following the meals. Blood samples were drawn at 20, 40, 60, 90 and 120 min following the meal and analysed for plasma glucose, serum insulin, and serum hs-CRP concentrations. Core body temperature was measured continuously for $120 \mathrm{~min}$ following the meal. The ingested CoreTemp $^{\mathrm{TM}}$ sensor telemetrically transmitted a signal to a portable data logger, which recorded and stored the temperature data (CorTemp ${ }^{\mathrm{TM}}$; CT2000). At the end of visit 2, participants were issued a second CorTemp ${ }^{\mathrm{TM}}$ capsule to be ingested prior to visit 3 .

Meals. The chicken burger meals used in this study were provided by one restaurant belonging to a South African chain. Both meals (HOT and CON) are commercially available and were cooked by the restaurant according to standard operating procedures, as they would for members of the public. As such, the HOT meal is available to the public as the 'Extra Hot' chicken burger option, and the CON meal is the 'Chilli-Free' option, where the standardised franchise recipe determines the capsaicin content in the meals. Both meals (HOT and CON) consisted of a chicken breast $( \pm 127.5 \mathrm{~g})$ served on a white bread roll, with lettuce and tomato garnish. The nutritional composition of both meals, as determined by Microchem Laboratory Services (Pty) Ltd, a South African National Accreditation System (SANAS)-accredited laboratory, is presented in Table 2. Although the meals were similar with regard to carbohydrate and protein content, the HOT meal contained more fat and as a result had a higher energy content.

Prior to cooking, all chicken breasts (HOT and CON meals) were infused with marinade (lemon, garlic and spice) containing a small amount of capsicum oleoresin $( \pm 0 \cdot 86 \mu \mathrm{g}$ capsaicin/ breast). The chicken for both meals (HOT and CON) was also marinated in a sauce for $24 \mathrm{~h}$ prior to cooking, which contained capsicum oleoresin, cayenne pepper and African bird's eye chilli, estimated to deliver $\pm 0 \cdot 3 \mathrm{mg}$ capsaicin per meal. The chicken was then basted in $20 \mathrm{ml}$ of a Prego sauce, which contained $\pm 0 \cdot 1 \mathrm{mg}$ capsaicin (from cayenne pepper). Finally, the HOT meal was cooked in $60 \mathrm{ml}$ of the restaurant's extra hot sauce, and the CON meal was cooked in $20 \mathrm{ml}$ of tomato sauce (ingredients for both sauces in Appendix 1). Thus, the key

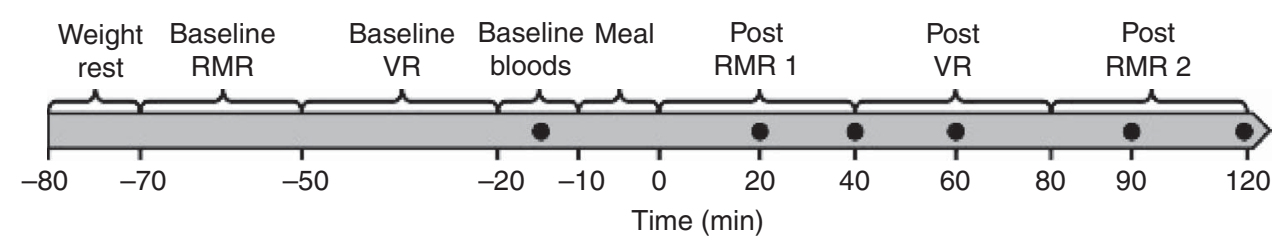

Fig. 1. Data collection timeline for visits 2 and 3. VR, endothelial-dependent microvascular reactivity test. 0 Time points at which blood was drawn to measure glucose, insulin and high sensitivity C-reactive protein concentrations. 
Table 2. Nutritional composition of the intervention (HOT) and control (CON) meals (per $280 \mathrm{~g}$ serving)

\begin{tabular}{lcc}
\hline & HOT $^{*}$ & CON† \\
\hline Energy (kJ) & 2295 & 1974 \\
Protein (g) & $47 \cdot 4$ & $47 \cdot 0$ \\
Carbohydrate (g) & $46 \cdot 2$ & $45 \cdot 4$ \\
Of which total sugar (g) & $8 \cdot 5$ & $10 \cdot 1$ \\
Total fat (g) & 20 & $10 \cdot 7$ \\
Of which saturated fat (g) & $4 \cdot 7$ & $3 \cdot 3$ \\
Of which trans fat (g) & $0 \cdot 0$ & $0 \cdot 0$ \\
Of which MUFA (g) & $5 \cdot 5$ & $3 \cdot 4$ \\
Of which PUFA (g) & $9 \cdot 7$ & $3 \cdot 9$ \\
Cholesterol (mg) & 73 & 70 \\
Dietary fibre (g) & $3 \cdot 1$ & $3 \cdot 1$ \\
$\mathrm{Na}^{+}$(mg) & 1080 & 912 \\
\hline
\end{tabular}

* Plain chicken burger with $60 \mathrm{ml}$ hot sauce.

† Plain chicken burger with $20 \mathrm{ml}$ tangy tomato sauce.

difference between the two meals was the sauce in which the chicken was cooked, and, by design, the amount of African bird's eye chilli delivered via each meal (Table 3).

As a means to determine the precise amount of capsaicin present in the HOT and CON meals and to monitor the consistency of the meal preparation during the study, capsaicin and total capsaicinoid levels from sample meals were analysed at an independent commercial laboratory using HPLC at the beginning and end of the study. The results of these analyses are presented in Table 3. On the basis of data averaged from the start and end analyses, there was approximately 5.82 and $<1.0 \mathrm{mg}$ of total capsaicinoids present in the HOT and CON meals, respectively, with capsaicin being the major component (HOT: $\pm 3.39 \mathrm{mg}$ and CON: $<0.69 \mathrm{mg}$ ).

Laboratory analyses. Venous blood samples were drawn prior to and 20, 40, 60, 90 and 120 min after ingesting the meal. Blood samples were collected into potassium oxalate and sodium fluoride vacutainer tubes for the determination of plasma glucose concentrations, lithium heparin vacutainer tubes for serum insulin concentrations and serum-separating vacutainer tubes containing a clot activator for hs-CRP serum concentration. All samples were kept on ice until centrifuged at $3200 \boldsymbol{g}$ at $4^{\circ} \mathrm{C}$ for $10 \mathrm{~min}$ upon completion of the test. Plasma samples were stored at $-20^{\circ} \mathrm{C}$ (glucose) or $-80^{\circ} \mathrm{C}$ (insulin and hs-CRP) until subsequent analyses. Plasma glucose concentrations were determined using the glucose oxidase method (YSI 2300 STAT PLUS). The intra-assay and interassay coefficients of variation for plasma glucose were 1.4 and $2.5 \%$, respectively. Serum insulin concentrations were determined using an automated chemiluminescence system (ACS-Centaur; Siemens Healthcare Diagnostics Inc.) with insulin reagent kits, calibrators and controls bought from the company. Intra-assay and interassay coefficients of variation for serum insulin were 2.4 and $4 \cdot 1 \%$, respectively. Serum hs-CRP was determined using rapid immunodiffusion at a medical diagnostic laboratory. The intraassay and interassay coefficients of variation for serum hs-CRP were 1.0 and $5.3 \%$, respectively.

Indirect calorimetry. Metabolic rates at rest and post-meal were determined using the ventilated hood technique with a
Table 3. Capsaicinoid analysis of the intervention (HOT) and control (CON) meals (absolute values)*

\begin{tabular}{lrrrrrr}
\hline & \multicolumn{2}{c}{ HOT } & & \multicolumn{2}{c}{ CON } \\
\cline { 2 - 3 } & Start & End & & Start & End \\
\hline Nordihydrocapsaicin $(\mathrm{mg} / \mathrm{kg})$ & $<1.0$ & $<1.5$ & & $<0.5$ & $<1.5$ \\
Capsaicin $(\mathrm{mg} / \mathrm{kg})$ & 14.7 & 13.0 & & 5.1 & $<1.5$ \\
Dihydrocapsaicin $(\mathrm{mg} / \mathrm{kg})$ & 9.6 & 8.0 & & $<0.5$ & $<1.5$ \\
Total capsaicinoids $(\mathrm{mg} / \mathrm{kg})$ & 24.3 & 23.0 & & 5.1 & $<4.5$ \\
Scoville heat index & 432 & 387 & & 92 & $<67$ \\
\hline
\end{tabular}

Start and end, beginning and end of the data collection phase of the study, respectively.

*Values approximated as '<' are lower than the limit of quantification for the method employed.

paramagnetic $\mathrm{O}_{2}$ analyser and infrared digital $\mathrm{CO}_{2}$ analyser (Cosmed CPET; Cosmed). RMR was calculated using Weir's formulae (Weir, 1949). The RMR test conducted during visit 1 served as a familiarisation test. Baseline RMR for each participant was taken as the measurement obtained prior to each meal during visits 2 and 3. Metabolic rate was measured with participants sitting quietly in a semi-recumbent position, whereas oxygen uptake and carbon dioxide production were recorded at $5 \mathrm{~s}$ intervals for either a $20 \mathrm{~min}$ period (baseline RMR) or two 40 min periods (post-meal metabolic rate). Data were averaged over $60 \mathrm{~s}$ intervals, and baseline RMR was taken as the mean value over the last $10 \mathrm{~min}$ of the baseline test, whereas postmeal metabolic rate was determined for 10 min intervals from immediately after until $2 \mathrm{~h}$ after the meal. The effect of the meal on metabolic rate was determined by comparing baseline RMR with metabolic rate measured after the meal (0-40 and 80-120 min).

To ensure accuracy, the gas analysers were calibrated before each trial was undertaken with a $3 \mathrm{~L}$ syringe and standard gas mixtures of oxygen and carbon dioxide $\left(4 \% \mathrm{CO}_{2}, 16 \% \mathrm{O}_{2}\right.$ and the balance nitrogen) (BOC Special Gas; Afrox) according to the manufacturer's instructions. On separate occasions once a month, the accuracy of the gas analysers was also validated against the combustion of $5 \mathrm{ml}$ of pure ethanol (97\%) for the duration of the research trial $( \pm 7$ months). The ethanol burns revealed mean errors of 0.7 (SD 0.5$) \%$ for RQ measurement and $0 \cdot 6(\mathrm{sD} 0 \cdot 5) \%$ for $\mathrm{CO}_{2}$ recovery ${ }^{(34)}$.

Endothelial-dependent microvascular reactivity. VR was measured non-invasively on the forearm, using laser Doppler imaging and iontophoresis of acetylcholine (ACh). Measurements were taken in a quiet, temperature-controlled room $\left(22-24^{\circ} \mathrm{C}\right)$, following $10 \mathrm{~min}$ of acclimatisation during baseline measurements, in the supine position. Briefly, the forearm was cleaned with an alcohol swab and excess alcohol removed with de-ionised water. Thereafter, an indifferent electrode was placed on the wrist, and adjacent to this an iontophoresis chamber was placed on the volar aspect of the same arm, using a double-sided adhesive ring. Three sites were used for the procedure: one site for the ACh vehicle ( $0.45 \%$ saline) and two neighbouring sites for the ACh drug ( $1 \% \mathrm{ACh}$, endothelium-dependent vasodilator). After filling the chamber with a solution, a battery-powered 
iontophoresis controller (MIC 2; Moor Instruments) provided a direct current for drug iontophoresis. Both ACh and ACh vehicle were delivered using an anodal current: 7 min of scanning time consisting of five $20 \mathrm{~s}$ pulses of $0.1 \mathrm{~mA}$ current, with a $60 \mathrm{~s}$ rest period between each pulse (total charge of $10 \mathrm{mC}$ ). To record erythrocyte flux, the laser Doppler imager (Moor LDI2; Moor Instruments Ltd; time constant $0 \cdot 1 \mathrm{~s}$, bandwidth $22 \mathrm{kHz}$ ) scanned the region of interest in twenty-one scans.

\section{Data and statistical analyses}

Data are presented as mean values and standard deviations. The homoeostasis model of assessment - insulin resistance was used to quantify insulin resistance and the Insulinogenic index to evaluate early-phase insulin secretion. AUC was determined using the trapezoidal method (GraphPad Prism, version 6.0b; GraphPad Software Inc.). Specifically, the AUC with respect to the ground method was employed ${ }^{(35)}$. The Shapiro-Wilks test was used to determine whether data were normally distributed. Sex comparisons were made using an independent $t$ test or the Mann-Whitney $U$ test. Baseline characteristics before the HOT and CON meals were compared using paired $t$ tests or Wilcoxon signed rank sum tests. Participant responses to each of the two meals were compared during the $2 \mathrm{~h}$ postprandial period using a two-way ANOVA with repeated measures for time and condition. A paired $t$ test was used to compare AUC data for variables measured after the HOT and CON meals. Statistical analyses were performed using STATA version 12 (StataCorp LP). Significance was accepted at $P<0 \cdot 05$.

(a)

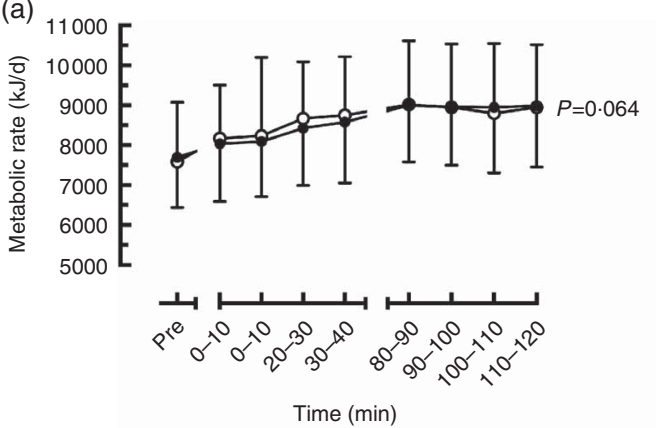

(c)

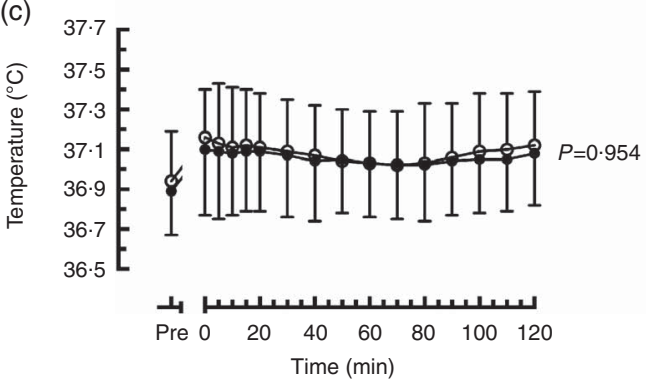

Results

\section{Participants}

All participants were overweight, by design, and had normal fasting glucose and insulin concentrations, although the women had higher resting insulin levels compared with the men ( $P=0.022$, Table 1$)$. On the basis of their OGTT responses, all but three could be described as having normal glucose tolerance (i.e. fasting plasma glucose $<5.6 \mathrm{mmol} / \mathrm{l}$ and $2 \mathrm{~h}$ post-OGTT glucose $<7.8 \mathrm{mmol} / \mathrm{l})^{(32)}$. Three participants (two females and one male) could be classified as having isolated abnormal fasting glucose concentrations (i.e. fasting plasma glucose between 5.6 and $7.0 \mathrm{mmol} / \mathrm{l}$ and $2 \mathrm{~h}$ post-OGTT glucose $<7.8 \mathrm{mmol} / \mathrm{l}$ ). The derived insulinogenic and Matsuda indices of the group were normal (Table 1).

\section{Baseline variables}

Participants weighed the same prior to eating the HOT and CON meals (HOT: 91.2 (sD 14.3) kg, CON: 91.3 (sD 14.3) kg, $P=0.284$ ) and had fasted for the same length of time (HOT: 12.9 (sD 1.5) h, CON: 13.2 (sD 1.5) h, $P=0 \cdot 146$ ). As can be seen from Fig. 2 and 3 and Table 3 (time point 'Pre'), there were also no differences in the baseline levels of RMR $(P=0.421)$, core temperature $(P=0.480)$, fasting glucose $(P=0.946)$, fasting insulin $(P=9.516)$, fasting hs-CRP $(P=0.973)$ and maximum VR $(P=0.398)$ measured prior to the HOT and CON meals. Although all but one participant had normal fasting glucose concentrations prior to both meals, mean fasting insulin concentrations were higher than normal $(>8.64 \mathrm{mU} / \mathrm{l})$ prior to both meals.

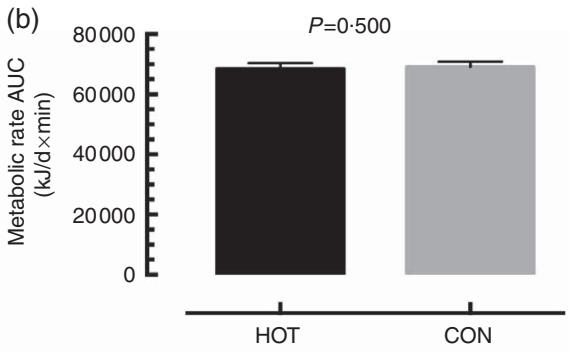

(d)

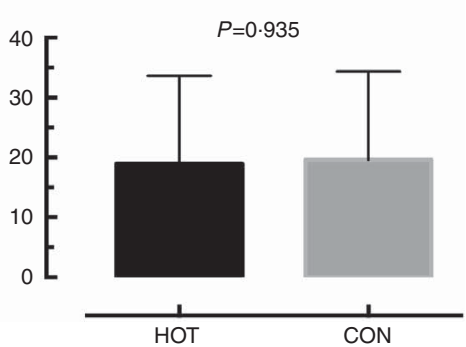

Fig. 2. Metabolic rate $(a, b)$ and core temperature $(c, d)$ responses to the intervention (HOT) and control (CON) meals. (a, c) Mean and standard deviation values measured at each time point from prior to (Pre) until the end of the $2 \mathrm{~h}$ postprandial period. (b, d) AUC values calculated for each variable measured during the same $2 \mathrm{~h}$ time period. Values are means and standard deviations. The $P$ values represent the time-by-condition interaction effect as determined using a two-way ANOVA with repeated measures for time and condition $(\mathrm{a}, \mathrm{c})$ and a paired $t$ test $(\mathrm{b}, \mathrm{d}) . \longrightarrow$ - $(\mathrm{a}, \mathrm{c}) \mathrm{HOT}$; —o—: (a, c) CON. 


\section{Meal effects}

The participants perceived the intervention meal to be hotter than the control meal (HOT: 8.3 (sD 1.7), CON: $0 \cdot 4$ (SD 0.8), $P<0.001)$. Fig. 2 shows the metabolic rate and core temperature responses of all participants to the HOT and CON meals. A two-way ANOVA with repeated measures for both time and condition showed no time-by-condition interaction effects in metabolic rate (a, $P=0.064)$ and core temperature (b, $P=0.954$ ), but both the HOT and CON meals did produce the expected thermic effect of feeding, as indicated by significant time effects (RMR: $P<0.001$, core temperature: $P<0.001)$. A paired $t$ test found no differences in calculated AUC for both RMR (b) and core temperature (d) in the $2 \mathrm{~h}$ postprandial period for both meals. In addition, the peak core temperature reached was similar following both meals (HOT: $37 \cdot 16(\text { sD } 0 \cdot 29)^{\circ} \mathrm{C}$, CON: $37 \cdot 18(\text { sD } 0 \cdot 29)^{\circ} \mathrm{C}, P=0 \cdot 633$ ).

The glucose and insulin responses of the participants to the two meals are shown in Fig. 3. Although there were no time-bycondition interaction effects for glucose $(P=0.298)$ and insulin $(P=0.855)$ following the HOT and CON meals (a, c), both variables displayed significant time effects (glucose: $P<0.001$ and insulin: $P<0.001)$. Further, a paired $t$ test found that the
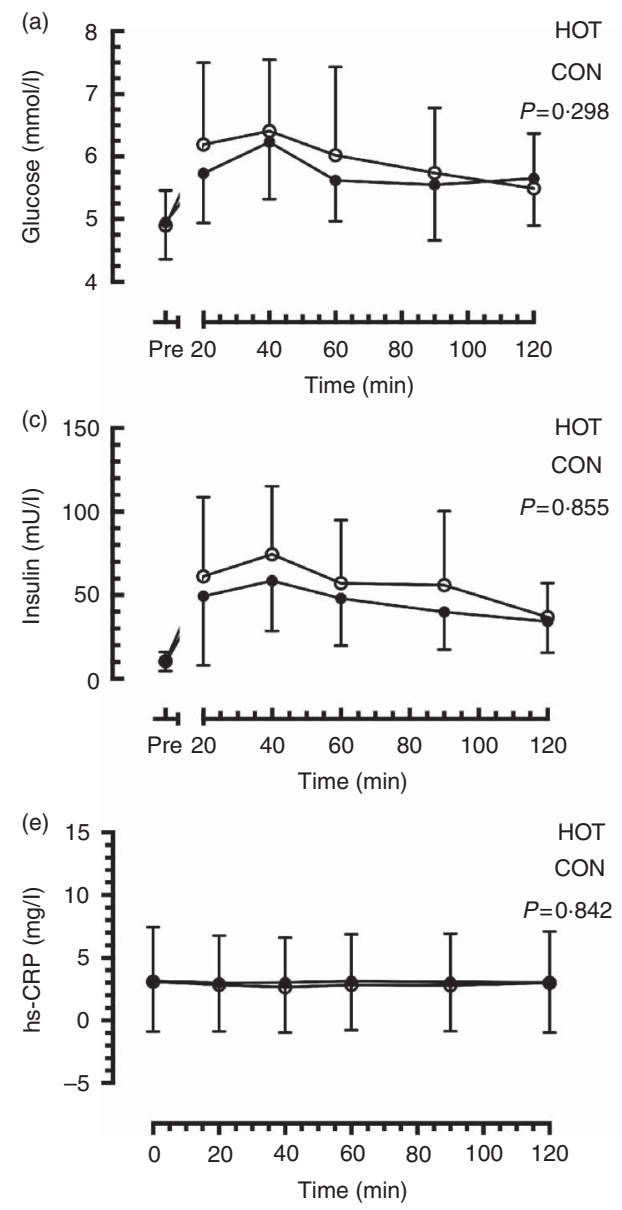

overall insulin AUC was lower when participants ate the HOT meal compared with the CON meal ( $d, P=0.002)$, and there was a tendency for glucose AUC to be lower following the HOT meal (b, $P=0.056)$.

There was no time-by-condition interaction effect for hs-CRP, and the related AUC was not different between the hot and control meals (Fig. 3(e) and (f)). Finally, there were no differences in any of the VR variables measured in response to the HOT and CON meals (Table 3). Given the differences in percentage body fat, fat-free mass and resting insulin concentrations between males and females (Table 1), the same analyses were repeated for males and females separately. However, the results were not different (data not shown).

\section{Discussion}

The main finding of this study was that $2 \mathrm{~h}$ postprandial insulin AUC was significantly lower in this group of overweight but non-diabetic individuals following the consumption of a commercially available meal containing chilli, in comparison with an otherwise similar meal with only trace amounts of capsaicin. Thus, despite the fact that the pattern of postprandial insulin
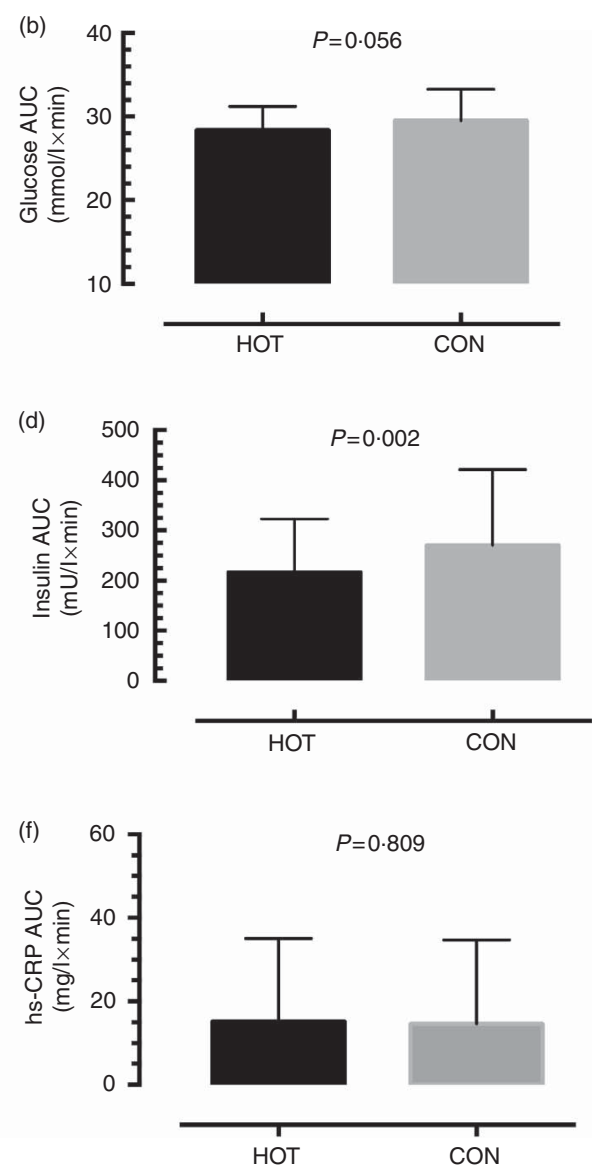

Fig. 3. Glucose (a, b), insulin (c, d) and high sensitivity C-reactive protein (hs-CRP) responses to the intervention (HOT) and control (CON) meals. (a, c, e) Mean and standard deviation values measured at each time point from prior to (Pre) until the end of the $2 \mathrm{~h}$ postprandial period. (b, $\mathrm{d}$, $\mathrm{f}$ ) AUC values calculated for each variable measured during the same $2 \mathrm{~h}$ time period. Values are means and standard deviations. The $P$ values represent the time-by-condition interaction effect as determined

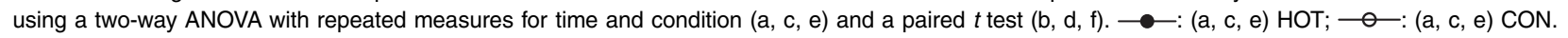


increase was similar following both meals, slightly less insulin was measured in the blood during this $2 \mathrm{~h}$ period following the HOT meal. These results support the work of Ahuja et al. ${ }^{(11)}$, who demonstrated attenuated increases in insulin following the consumption of a standardised meal seasoned with cayenne pepper. As the plasma glucose concentrations, measured for $2 \mathrm{~h}$ after ingestion of the meals, were not significantly different, the authors suggest that glucose disposal occurred similarly with lower insulin concentrations, implying increased insulin sensitivity after consumption of the HOT meal ${ }^{(11)}$. This finding is also in agreement with the earlier work of Chaiyata et al. ${ }^{(22)}$, who found that the ingestion of $5 \mathrm{~g}$ of fresh chilli pepper (Capsicum frutesens) attenuated the rise in plasma glucose for 30 min after the ingestion of a glucose-containing drink. What makes this study unique, however, is that the meal provided is a standard restaurant meal available to the public, containing a relatively small amount of chilli: $3.39 \mathrm{mg}$ of capsaicin, compared with an estimated $33 \mathrm{mg}$ capsaicin from $30 \mathrm{~g}$ cayenne chilli mix by Ahuja et al. $^{(11)}$ and approximately $37 \mathrm{mg}$ capsaicin from $5 \mathrm{~g}$ fresh chilli by Chaiyata et $a l^{(22)}$. The results are, however, in contrast to those of Domotor et al. ${ }^{(36)}$, who found no difference in plasma insulin concentrations, but reduced serum glucose and glucagon concentrations, when either capsaicin or a placebo was administrated prior to glucose loading. It should be noted, however, that the dosage of capsaicin administered in this study was very low $(0.4 \mathrm{mg})$.

The precise mechanisms through which capsaicin may attenuate postprandial hyperinsulinaemia are unclear. One potential mechanism may be through the effect of capsaicin on adiponectin, a hormone secreted by adipose tissue and thought to impact insulin sensitivity and glucose regulation. In mice given dietary capsaicin, higher concentrations of plasma adiponectin, adiponectin mRNA in adipose tissue and the adiponectin receptor were observed in parallel with lower resting concentrations of glucose, insulin and $\mathrm{TAG}^{(17)}$. Similar studies are yet to be conducted in humans. A second mechanism may be related to the influence that capsaicin may have on the gastrointestinal tract (GIT), given that the GIT is innervated with capsaicin-sensitive primary afferent sensory nerves ${ }^{(37)}$. Chilli ingestion may affect postprandial blood insulin concentrations via rate of gastric emptying, as Horowitz et $a l .{ }^{(38)}$ have shown that insulin response and gastric-emptying time are inversely related. The studies that have measured the rate of gastric emptying following chilli ingestion have, however, produced opposing results. Some have shown that chilli ingestion delays gastric-emptying time and quickens whole-gut transit time ${ }^{(39)}$, whereas others have shown an increase in gastric-emptying time after the ingestion of chilli ${ }^{(37)}$. The equivocal results from studies on insulin response and gastric emptying may be attributed to different methodological approaches in time, quantity and method of capsaicin administration. Finally, Ahuja et $a l^{(11)}$ speculate that a third potential mechanism may be through chilli increasing the number or affinity of hepatic insulin receptors, thereby increasing insulin clearance. Further research is needed to understand the possible mechanisms at molecular and/ or receptor levels that may explain the beneficial effects of capsaicin on insulin metabolism in humans.
The second finding of this study was that, although both the HOT and CON meals generated the expected thermic effect of feeding for the $2 \mathrm{~h}$ after ingestion, repeated-measures analyses revealed no quantifiable increase in either postprandial energy expenditure or core temperature attributable to the chillicontaining meal. These findings are in contrast to other studies ${ }^{(26)}$ and in particular to the earlier work of Chaiyata et $a l^{(22)}$, who showed that RMR immediately increased and remained elevated for $30 \mathrm{~min}$ after ingestion of $5 \mathrm{~g}$ of fresh chilli pepper. A recent review suggests that elevations in RMR may only occur at higher doses of capsaicin ${ }^{(40)}$. This would make sense in our case where the capsaicin content of the meal was only one-tenth of that administered by Chaiyata $e t a l .{ }^{(22)}$, further motivating a dose-response effect. Another point to consider would be the extent to which the participants habitually eat chilli. Ludy et al. ${ }^{(21)}$ suggest that habitual chilli consumers may become desensitised to the effects of chilli and as such show different metabolic rate responses compared with nonaccustomed chilli eaters.

The third finding of this study was that the chilli meal did not produce an effect on the VR response of the participants. Although the topical application of capsaicin has been shown to induce vasodilation ${ }^{(41)}$, there are limited studies investigating its consumption and relationship with microvascular function. One group has compared the effects of a 4 -week chilli-free (bland) $v$. chilli-supplemented (hot) diet on endothelium-independent and endothelium-dependent vasodilation in non-obese individuals, but found no differences in vascular responses between the two diets $^{(12)}$. Our study differed as it measured the acute effect of capsaicin consumption on the microcirculation of overweight and obese individuals as opposed to vascular (arterial) changes in a non-obese group. We may have missed a postprandial effect on microvascular reactivity because of the small sample size; however, the sub-study within the Ahuja et al. ${ }^{(12)}$ study had a comparable sample size and similarly found no endothelial-dependent vascular effect of capsaicin. Another possible explanation for our findings was the time frame in which measurements for microvascular reactivity were taken. Postprandial differences may have occurred at a later time point to that chosen for this study. In a number of studies measuring the acute postprandial effect of a testing ingredient such as dark chocolate $^{(42)}$, tea ${ }^{(43,44)}$, or beer ${ }^{(45)}$, measurement of endothelial function continued for at least $1 \mathrm{~h}$ after consumption. In contrast, our protocol only allowed for a single postprandial measurement 40 min after meal consumption. George et al. ${ }^{(46)}$, who measured microvascular function using a similar technique, found the greatest effect on microvascular function $7.5 \mathrm{~h}$ after consumption of a fruit and vegetable puree-based drink. This suggests that it is very likely that our postprandial testing time may have been insufficient to observe any effect.

A number of explanations exist for the discrepancies between this study and others. One may be the method of ingestion. Some studies have provided capsaicin in capsule ${ }^{(25)}$ form, whereas others have used chilli-containing meals ${ }^{(11,12,22)}$. There are benefits and limitations to both forms. Using capsule ingestion allows participants to be blinded in a controlled placebo trial. However, there is reason to believe that the burning sensation in the mouth experienced by eating chilli in 
Table 4. Endothelial-dependent microvascular reactivity (VR) parameters in response to the intervention (HOT) and control (CON) meals ${ }^{\star}$ (Mean values and standard deviations)

\begin{tabular}{|c|c|c|c|c|c|c|c|c|c|}
\hline & \multicolumn{4}{|c|}{ НOT } & \multicolumn{4}{|c|}{ CON } & \multirow[b]{3}{*}{$P+$} \\
\hline & \multicolumn{2}{|c|}{ Pre $(n 17)$} & \multicolumn{2}{|c|}{ Post (n 14) } & \multicolumn{2}{|c|}{ Pre $(n 13)$} & \multicolumn{2}{|c|}{ Post (n 13) } & \\
\hline & Mean & SD & Mean & SD & Mean & SD & Mean & SD & \\
\hline VR maximum $\ddagger$ & $370 \cdot 7$ & 153.5 & $380 \cdot 1$ & $154 \cdot 2$ & 363.5 & $104 \cdot 2$ & 345.9 & 109.9 & 0.610 \\
\hline VR \% change & 504.0 & 251.3 & 514.0 & 241.2 & 533.5 & 221.0 & $505 \cdot 0$ & 243.6 & 0.692 \\
\hline VR AUC & 68858 & 40274 & 76404 & 44107 & 64467 & 26371 & 58739 & 27691 & 0.348 \\
\hline
\end{tabular}

* The pre values represent the baseline measurement prior to eating the meals and the post values represent the measurement taken in the period $30-60$ min after eating the meal. $\dagger$ The $P$ value represents significance as determined by a two-way ANOVA with repeated measures for time and condition.

$\ddagger$ Absolute perfusion given in perfusion units.

food may be a necessary catalyst for subsequent responses. A study by Ludy et $a l^{(21)}$ showed that the change in energy expenditure was higher following ingestion of a meal containing red pepper than when the pepper was ingested in a capsule. Therefore, it is possible that the subsequent metabolic effects of capsaicin ingestion rely on signals induced in part by the initial burning experienced in the mouth, and that a capsule may blunt these responses. Potentially, this signalling may be mediated through inflammation. Capsaicin is an irritant that elicits a burning sensation when it comes into contact with mammalian tissue. It binds to the transient receptor potential variant 1 in sensory nerve fibre endings, resulting in the release of inflammatory neuropeptides ${ }^{(47)}$. This study found no evidence of systemic inflammation following ingestion of the chilli meal, because no differences in plasma C-reactive protein concentrations were observed between the HOT and CON meals. Alternatively, capsaicin stimulation of receptors in the mouth may trigger an anticipatory response in the brain, capable of modifying cortical nociceptive network activation, because a study by Porro et al. ${ }^{(48)}$ has shown the involvement of both the sensory and affective components of pain, even in the absence of actual noxious input. Although branches of the glossopharyngeal and vagus nerves that innervate the pharynx, and perhaps the larynx, appear to respond more strongly to ingested capsaicin than do the branches of the trigeminal nerve that innervate the oral mucosa ${ }^{(49)}$, further research is warranted to fully understand the brain's response to capsaicin ingestion.

Studies have also differed with respect to the active ingredient used. Some have provided capsiate - a capsinoid ${ }^{(25)}$ - whereas others use the more active capsaicin - a capsaicinoid ${ }^{(11,12)}$. Capsinoids, which are also found in chillies, are structurally similar to capsaicinoids, but are only about one-thousandth as pungent. Another difference may be the dosage of capsaicin delivered. Of the studies that have assessed the effects of capsaicin specifically, the reported capsaicin values ingested varied from 1 to $140 \mathrm{mg}^{(7-13,21-23)}$. A final difference to be considered is the study population. For example, the extent to which participants habitually eat chilli may also influence the outcome, as regular chilli users may become desensitised to the effects of capsaicin ${ }^{(21)}$.

One potential limitation of this study is that of sample size. As this study was similar in design to that of Ahuja et al. ${ }^{(11)}$, we based our sample size on their data, which showed that for their thirty-six participants a significant difference $( \pm 20 \%)$ in postprandial insulin AUC between hot and control meals was observed. On the basis of this study and that of Ahuja et al. ${ }^{(11)}$, it seems that this sample size is adequate to detect differences in $2 \mathrm{~h}$ postprandial insulin AUC. Looking more closely at the repeatedmeasures data for metabolic rate (Fig. 2(a)), temperature (Fig. 2 (c)), glucose (Fig. 3(a)), insulin (Fig. 3(c)) and VR (Table 4), however, one observes large differences in inter-individual variation, as indicated by the large standard deviations. Retrospective power analyses indicated that power was very low for all these variables (data not shown). Therefore, it is recommended that, in the future, larger sample sizes might be better able to detect potential time-by-treatment interactions for these variables.

A second limitation is that the total energy and fat content of the HOT meal was higher than that of the CON meal (Table 2). Although one cannot exclude this as a factor potentially explaining the lower $2 \mathrm{~h}$ postprandial insulin response for the HOT meal, it seems unlikely. Rather, there is evidence to suggest that, although fat alters the gastric-emptying rate, it does not reduce the postprandial insulin response ${ }^{(50)}$. Finally, as the potential interactions between capsaicin and fat are unknown, this could be addressed in the future.

\section{Conclusion}

The results from this study indicate that a standard restaurant meal containing a relatively small dose of capsaicin delivered via African bird's eye chilli, which is currently available to the public, results in lower postprandial insulin concentrations in overweight individuals, compared with the same meal without chilli. This is the first such study to show this effect with a small and arguably palatable dose of chilli. Therefore, we cautiously speculate that there may be potential health benefits related to chilli consumption on postprandial glucose control, and that this may be of particular relevance to pre-diabetic and/or diabetic individuals. However, it is premature to make definite claims along these lines, and future research is needed to address the following issues - for example, do the acute effects observed in this study persist with chronic use of chilli? Or is the effect dampened or lost in accustomed chilli eaters? How long do the effects last for, and how often would chilli need to be consumed? Are similar effects observed in normal weight individuals? And what about the diabetic population? 


\section{Acknowledgements}

Thanks to Hendriena Victor, Liske Kotze, Sharief Hendricks, Melanie Hay and Veeraj Goyaram for assistance with data collection and laboratory analysis.

This research was requested by and funded in part by Nando's South Africa, a restaurant chain that serves the meals tested in this study. Nando's South Africa had no role in the design, analysis or writing of this article. This study was also funded in part by a grant from the National Research Foundation of South Africa's Technology and Human Resources for Industry Programme (UID80029). The UCT/MRC Research Unit for Exercise Science and Sports Medicine is funded in part by Discovery Vitality and the South African Medical Research Council.

J. K. - data acquisition, data analysis and interpretation, manuscript drafting and revision, final approval of manuscript; D. H. - data acquisition, data interpretation, manuscript drafting and revision, final approval of manuscript; P. P. - data acquisition, data analysis and interpretation, manuscript drafting and revision, final approval of manuscript; R. T. - conception and design, data interpretation, manuscript revision, final approval of manuscript; V. L. - conception and design, data interpretation, manuscript revision, final approval of manuscript; D. R. conception and design, data acquisition, data analysis and interpretation, manuscript drafting and revision, final approval of manuscript.

There are no conflicts of interest to declare.

\section{References}

1. Butt MS \& Sultan MT (2011) Coffee and its consumption: benefits and risks. Crit Rev Food Sci 51, 363-373.

2. Murphy EA, Davis JM, Carmichael MD, et al. (2009) Benefits of oat -glucan and sucrose feedings on infection and macrophage antiviral resistance following exercise stress. Am J Physiol Regul Integr Comp Physiol 297, R1188-R1194.

3. Berry NM, Davison K, Coates AM, et al. (2010) Impact of cocoa flavanol consumption on blood pressure responsiveness to exercise. Br J Nutr 103, 1480-1484.

4. Fraga C, Litterio M, Prince P, et al. (2010) Cocoa flavanols: effects on vascular nitric oxide and blood pressure. Clin J Biochem Nutr 48, 63-67.

5. Mustafa RA, Hamid AA, Mohamed S, et al. (2010) Total phenolic compounds, flavonoids, and radical scavenging activity of 21 selected tropical plants. J Food Sci 75, C28-C35.

6. Galleano M, Pechanova O \& Fraga CG (2010) Hypertension, nitric oxide, oxidants, and dietary plant polyphenols. Curr Pharm Biotechnol 11, 837-848.

7. Reinbach HC, Smeets A, Martinussen T, et al. (2009) Effects of capsaicin, green tea and $\mathrm{CH}-19$ sweet pepper on appetite and energy intake in humans in negative and positive energy balance. Clin Nutr 28, 260-265.

8. Smeets AJ \& Westerterp-Plantenga MS (2009) The acute effects of a lunch containing capsaicin on energy and substrate utilisation, hormones, and satiety. Eur J Nutr 48, 229-234.

9. Chaiyasit K, Khovidhunkit W \& Wittayalertpanya S (2009) Pharmacokinetic and the effect of capsaicin in Capsicum frutescens on decreasing plasma glucose level. J Med Assoc Thai 92, 108-113.

10. Ericson A, Nur EM, Petersson F, et al. (2008) The effects of capsaicin on gastrin secretion in isolated human antral glands: before and after ingestion of red chilli. Digest Dis Sci 54, 491-498.
11. Ahuja KD, Robertson IK, Geraghty DP, et al. (2006) Effects of chili consumption on postprandial glucose, insulin, and energy metabolism. Am J Clin Nutr 84, 63-69.

12. Ahuja KDK, Robertson IK, Geraghty DP, et al. (2007) The effect of 4-week chilli supplementation on metabolic and arterial function in humans. Eur J Clin Nutr 61, 326-333.

13. Nelson AG, Glickman-Weiss E \& Day R (2000) The effect of capsaicin on the thermal and metabolic responses of men exposed to 38 degrees $\mathrm{C}$ for 120 minutes. Wilderness Environ Med 11, 152-156.

14. Watanabe T, Kawada T, Yamamoto M, et al. (1987) Capsaicin, a pungent principle of hot red pepper, evokes catecholamine secretion from the adrenal medulla of anesthetized rats. Biochem Biophys Res Commun 142, 259-264.

15. Kawada T, Watanabe T, Takaishi T, et al. (1986) Capsaicininduced beta-adrenergic action on energy metabolism in rats: influence of capsaicin on oxygen consumption, the RQ, and substrate utilization. Proc Soc Exp Biol Med 183, 250-256.

16. Kang J-H, Goto T, Han I-S, et al. (2010) Dietary capsaicin reduces obesity-induced insulin resistance and hepatic steatosis in obese mice fed a high-fat diet. Obesity 18, 780-787.

17. Kang J-H, Tsuyoshi G, Le Ngoc H, et al. (2011) Dietary capsaicin attenuates metabolic dysregulation in genetically obese diabetic mice. J Med Food 14, 310-315.

18. Lejeune MPGM, Kovacs EMR \& Westerterp-Plantenga MS (2007) Effect of capsaicin on substrate oxidation and weight maintenance after modest body weight loss in human subjects. BrJ Nutr $\mathbf{9 0}, 651$.

19. Janssens PLHR, Hursel R, Martens EAP, et al. (2013) Acute effects of capsaicin on energy expenditure and fat oxidation in negative energy balance. PLOS ONE 8, e67786.

20. Smeets AJ, Janssens PLHR \& Westerterp-Plantenga MS (2013) Addition of capsaicin and exchange of carbohydrate with protein counteract energy intake restriction effects on fullness and energy expenditure. $J$ Nutr 143, 442-447.

21. Ludy M-J \& Mattes RD (2011) The effects of hedonically acceptable red pepper doses on thermogenesis and appetite. Physiol Behav 102, 251-258.

22. Chaiyata P, Puttadechakum S \& Komindr S (2003) Effect of chili pepper (Capsicum frutescens) ingestion on plasma glucose response and metabolic rate in Thai women. $J$ Med Assoc Thai 86, 854-860.

23. Lim K, Yoshioka M, Kikuzato S, et al. (1997) Dietary red pepper ingestion increases carbohydrate oxidation at rest and during exercise in runners. Med Sci Sport Exerc 29, 355-361.

24. Yoshioka M, St-Pierre S, Suzuki M, et al. (1998) Effects of red pepper added to high-fat and high-carbohydrate meals on energy metabolism and substrate utilization in Japanese women. Br J Nutr 80, 503-510.

25. Inoue $\mathrm{N}$, Matsunaga $\mathrm{Y}$, Satoh $\mathrm{H}$, et al. (2007) Enhanced energy expenditure and fat oxidation in humans with high BMI scores by the ingestion of novel and non-pungent capsaicin analogues (capsinoids). Biosci Biotechnol Biochem 71, 380-389.

26. Whiting S, Derbyshire E \& Tiwari BK (2012) Capsaicinoids and capsinoids. A potential role for weight management? A systematic review of the evidence. Appetite 59, 341-348.

27. Westerterp-Plantenga MS \& Smeets A (2005) Lejeune MPG. Sensory and gastrointestinal satiety effects of capsaicin on food intake. Int J Obes (Lond) 29, 682-688.

28. Eringa EC, Serne EH, Meijer RI, et al. (2013) Endothelial dysfunction in (pre)diabetes: characteristics, causative mechanisms and pathogenic role in type 2 diabetes. Rev Endocrinol Metab Disord 14, 39-48.

29. Muniyappa R \& Sowers JR (2013) Role of insulin resistance in endothelial dysfunction. Rev Endocrinol Metab Disord 14, 5-12. 
30. Appel SJ, Jones ED \& Kennedy-Malone L (2004) Central obesity and the metabolic syndrome: implications for primary care providers. J Am Acad Nurse Pract 16, 335-342.

31. Kawada T, Otsuka T, Endo T \& Kon Y (2011) Prevalence of the metabolic syndrome and its relationship with diabetes mellitus by aging. Aging Male 14, 203-206.

32. Genuth S, Alberti KGMM, Bennett P, et al. (2003) Follow-up report on the diagnosis of diabetes mellitus. Diabetes Care 26, 3160-3167.

33. Lean ME, Han TS \& Morrison CE (1995) Waist circumference as a measure for indicating need for weight management. Br Med J 311, 158-161

34. Blond E, Maitrepierre C, Normand S, et al. (2011) A new indirect calorimeter is accurate and reliable for measuring basal energy expenditure, thermic effect of food and substrate oxidation in obese and healthy subjects. Eur e-J Clin Nutr Metab 6, e7-e15.

35. Pruessner JC, Kirschbaum C, Meinlschmid G, et al. (2003) Two formulas for computation of the area under the curve represent measures of total hormone concentration versus timedependent change. Psychoneuroendocrinology 28, 916-931.

36. Dömötör A, Szolcsányi J \& Mózsik G (2006) Capsaicin and glucose absorption and utilization in healthy human subjects. Eur J Pharmacol 534, 280-283.

37. Debreceni A, Abdel-Salam OM, Figler M, et al. (1999) Capsaicin increases gastric emptying rate in healthy human subjects measured by 13C-labeled octanoic acid breath test. J Physiol Paris 93, 455-460.

38. Horowitz M, Edelbroek MA, Wishart JM, et al. (1993) Relationship between oral glucose tolerance and gastric emptying in normal healthy subjects. Diabetologia 36, 857-862.

39. Horowitz M, Wishart J, Maddox A, et al. (1992) The effect of chilli on gastrointestinal transit. J Gastroenterol Hepatol 7, 52-56.

40. Mattes RD (2012) Spices and energy balance. Physiol Behav 107, 584-590.

41. Van der Schueren BJ, de Hoon JN, Vanmolkot FH, et al. (2007) Reproducibility of the capsaicin-induced dermal blood flow response as assessed by laser Doppler perfusion imaging. $\mathrm{BrJ}$ Clin Pharmacol 64, 580-590.

42. Faridi Z, Njike VY, Dutta S, et al. (2008) Acute dark chocolate and cocoa ingestion and endothelial function: a randomized controlled crossover trial. Am J Clin Nutr 88, 58-63.
43. Alexopoulos N, Vlachopoulos C, Aznaouridis K, et al. (2008) The acute effect of green tea consumption on endothelial function in healthy individuals. Eur J Cardiovasc Prev Rehabil 15, 300-305.

44. Hodgson JM, Burke V \& Puddey IB (2005) Acute effects of tea on fasting and postprandial vascular function and blood pressure in humans. J Hypertens $\mathbf{2 3}, 47-54$

45. Karatzi K, Rontoyanni VG, Protogerou AD, et al. (2013) Acute effects of beer on endothelial function and hemodynamics: a single-blind, crossover study in healthy volunteers. Nutrition 29, 1122-1126.

46. George TW, Paterson E, Waroonphan S, et al. (2012) Effects of chronic consumption of fruit and vegetable puree-based drinks on vasodilation, plasma oxidative stability and antioxidant status. J Hum Nutr Diet 25, 477-487.

47. Hayman M \& Kam PCA (2008) Capsaicin: a review of its pharmacology and clinical applications. Curr Anaesth Crit Care 19, 338-343.

48. Porro CA, Baraldi P, Pagnoni G, et al. (2002) Does anticipation of pain affect cortical nociceptive systems? J Neurosci 22, 3206-3214.

49. Rentmeister-Bryant H \& Green BG (1997) Perceived irritation during ingestion of capsaicin or piperine: comparison of trigeminal and non-trigeminal areas. Chem Senses 22, 257-266.

50. Collier G, McLean A \& O'Dea K (1984) Effect of co-ingestion of fat on the metabolic responses to slowly and rapidly absorbed carbohydrates. Diabetologia 26, 50-54.

\section{Appendix 1: Sauce ingredients}

HOT meal 'extra hot sauce'

Water, vinegar, salt, lemon, African bird's eye chilli, onion, cayenne pepper, vegetable oil, dehydrated green pepper, paprika, garlic, thickener and stabilisers.

\section{CON meal 'tangy tomato sauce'}

Water, tomato paste, garlic purée, onion purée, sugar, lemon concentrate, lime concentrate, molasses, cayenne pepper, African bird's eye chilli and stabilisers. 